\title{
Friction compensation in contouring control for biaxial feed drive systems and experimental verification
}

\author{
Dinh Ba BUI, Naoki UCHIYAMA ${ }^{1}$ and Shigenori SANO \\ ${ }^{1}$ Department of Mechanical Engineering, Toyohashi University of Technology, Japan, uchiyama@tut.jp
}

\begin{abstract}
:
Friction is a primary disturbance in mechanical motion control of high precision machines. Because the friction causes undesired effects such as steady state and tracking motion errors of industrial machines, control systems should be designed to compensate for frictional effects. In this paper, we propose a simple yet really efficient method for estimating the friction property in biaxial feed drive systems. Our method employs the conventional circular contouring control results in estimating the friction property for contouring controller design. The effectiveness of the proposed method is demonstrated by experimental results for different contour curves in different motion speeds.
\end{abstract}

Keywords: Machine tool, Friction compensation, Biaxial feed drive dynamics, Contouring control, High-precision machining.

\section{Introduction}

In machine tool control, position error with respect to each feed drive axis is generally used as feedback signals. However, regarding to machining, error components orthogonal to the desired contour curves are more important than errors regarding to the feed drive axes. Error components orthogonal to the contour curves are called contour errors. There are many controllers proposed for reducing contour error and its efficiency has been proved by experiment.

Among the many proposals for reducing contour error [1], Koren proposed a cross-coupling controller that calculated contour error from drive axis tracking error and reduced contour error [2]. Advanced optimal and nonlinear controllers applied to contouring controller design have had their effectiveness verified [3][4], although they have disadvantage that both contour and tracking errors in a feed drive axis are used to calculate control input, and this compromise contour tracking performance. Considering contour and tracking errors simultaneously, creates difficulties in adjusting controller parameters.

Lo and Chung proposed contouring control for biaxial feed drives based on a coordinate transformation [5], in which feed drive axis tracking error is converted to error components orthogonal and tangential to the desired contour curve. This obtains two decoupled single input/output systems for the orthogonal and tangential directions, and enables controllers for each direction to be designed independently, and hence simplifies controller parameter adjustment. Chiu and Tomizuka proposed a similar approach, whereby a task coordinate frame is defined at the desired position of the feed drive system, and the control system dynamics are reformulated with respect to this coordinate frame correspond to the orthogonal and tangential directions of the desired contour curve, and the orthogonal error component is regarded as an approximation of the actual contour error
[6]. Although this approach provides control system stability for any contour curve, the difference between the actual contour error and the determined orthogonal error component may result in an increase in the contour error. A similar approach for feed drive system with fourth-order dynamics has been presented in [7].

In this paper, we improve the contouring controller design for biaxial feed drive systems developed by one of the authors [8] by including the friction compensation. Many friction models have been proposed thus far, and can be divided into two categories: kinetic and dynamic friction models. The kinetic friction models take into account major friction effects such as the viscous friction, the Coulomb friction and the Stribeck effect [9]. The dynamic friction models such as the LuGre model consider the natural mechanism of friction generation [10]. Although there have been many sophisticated fiction models, we verify the contouring control performance by using a simple fiction model consisting of the viscous and the Coulomb ones to clarify its effectiveness and limitations. A contouring control result based on a typical circular motion is used for the estimation of friction parameters. The control input is divided into two terms: a nominal dynamics term and a control term. Considering only the control term, we can estimate the friction parameters in a simple manner. Based on the estimated friction parameters, we include a simple friction compensator in a contouring controller. By applying this compensator, the control input profile coincides with the nominal dynamics term in repetitive circular motion experiments, and more than $70 \%$ reduction of the contour error is achieved on average. Applying the designed friction compensator with the same friction parameters to several non-circular motion contours in the experiment, we confirm that the effectiveness is maintained and around $70 \%$ reduction of the contour error is achieved as well. 


\section{Feed drive dynamics}

2.1 Definitions of tracking errors and contouring control

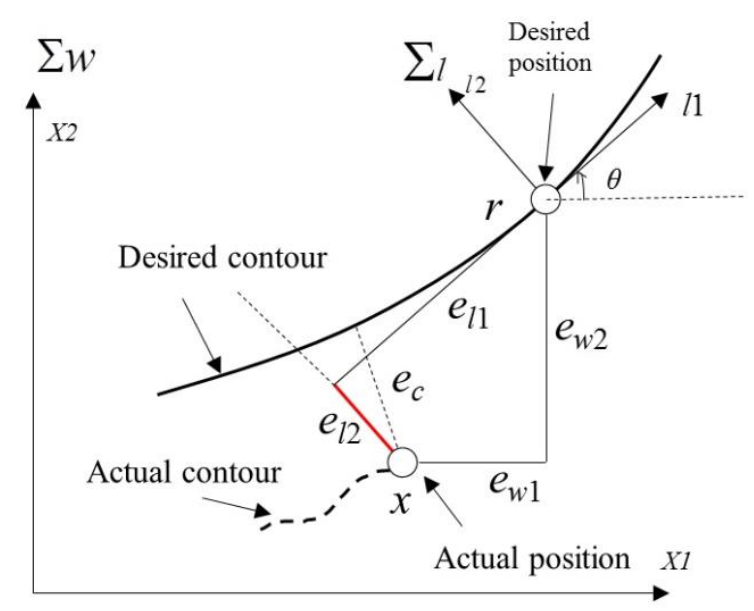

Fig. 1: Definition of contour error.

Fig. 1 schematically explains the relation between the tracking errors in each feed drive axis and the contour error. The coordinate frame $\sum_{w}$, whose axes $X_{1}$ and $X_{2}$ correspond to the feed drive axes, is a fixed frame. The curve $c$ in the figure is the desired contour curve of the point of a machined part driven by the feed drive system. The symbol $r=\left[r_{1}, r_{2}\right]^{T}$ is the desired position of the point of the machined part at time $t$, and defined in $\sum_{w}$. The actual position of the feed drive system is assumed to be $\mathrm{x}=\left[\mathrm{x}_{1}, \mathrm{x}_{2}\right]^{\mathrm{T}}$, which is also defined in $\sum_{w}$. A local coordinate frame $\sum_{l}$ is also defined, its origin is at $r$ and axes are $\sum_{l i}(\mathrm{i}=1,2)$, as shown in the figure. The axis $\sum_{l 1}$ is in the tangential direction of $c$ at $r$, and the direction of $l_{2}$ is perpendicular to $l_{l}$. The tracking error vector $e_{w}$, which consists of the tracking errors of both feed drive axes, is defined as follows:

$$
E_{w}=\left[e_{w 1}, e_{w 2}\right]^{T}=x-r
$$

This error vector can be expressed with respect to $\sum_{l}$ as follows:

$$
\begin{gathered}
E_{l}=\left[e_{l 1}, e_{l 2}\right]^{T}=R^{T} e_{w} \\
R^{T}=\left[\begin{array}{cc}
\cos \theta & -\sin \theta \\
\sin \theta & \cos \theta
\end{array}\right]
\end{gathered}
$$

where the inclination $\theta$ of $\sum_{l}$ to $\sum_{w}$ is as shown in Fig. 1. Regard to machining, error component orthogonal to the desired contour curve are more significant than the tracking errors of the feed drive axes. Thus, a controller design is proposed that allows the control performance to be adjusted with respect to each axis of $\sum_{l}$ independently for feed drive systems with fourth-order dynamics [8].

Because the error component tangential to the contour curve is less significant than the orthogonal one, the controller gain should be set for reducing the error along $l_{1}$ to lower than the gain for the error along $l_{2}$, and the proposed approach allows such gain assignment.

\subsection{Feed drive dynamics}

The controller design presented here for biaxial feed drives driven by servomotors, commonly used in industrial applications, involves feed drive dynamics represented by the following decoupled second-order system:

$$
\begin{aligned}
& M \ddot{x}+C \dot{x}=v \\
& M=\operatorname{diag}\left\{m_{i}\right\}, C=\operatorname{diag}\left\{c_{i}\right\}, i=1,2, \\
& v=\left[v_{1}, v_{2}\right]^{T},
\end{aligned}
$$

where $m_{i}(>0), c_{i}(\geq 0)$ and $v_{i}$ are the table mass, viscous friction coefficient and driving force on the drive axis $i$, respectively. The symbol $\operatorname{diag}\left\{a_{i}\right\}$ denotes a diagonal matrix with elements $a_{i}$ at the $i$ th diagonal positions.

\section{Friction compensation}

This section presents a coordinate transformation-based contouring controller design with friction compensation. The following items are assumed for the controller design:

1. The desired trajectory $r_{i}$ and its derivative $\dot{r}_{l}$ and $\ddot{r}_{l}$ are available.

2. The inclination of the local coordinate frame $\sum_{l}$ in Fig. 1 is available.

3. The position of the feed drive system $x$ and its derivative $\dot{x}$ are measurable.

4. Feed drive dynamics parameter values for $m_{i}$ and $c_{i}$ are available.

Under these assumptions and from reference [8], we have the following control:

$$
\begin{gathered}
v=M\left\{\ddot{r}-R\left(K_{v l} \dot{e}_{l}+K_{p l} e_{l}\right)-\ddot{\theta} I_{e} e_{w}+\dot{\theta}^{2} I e_{w}-\right. \\
\left.2 \dot{\theta} I_{e} e_{w}\right\}+C \dot{x} \\
I_{e}=\left[\begin{array}{cc}
0 & 1 \\
-1 & 0
\end{array}\right]
\end{gathered}
$$

where $I$ and $\ddot{r}$ are $2 \times 2$ identity matrix and reference acceleration of desired contour, respectively. The symbols $K_{v l}$ and $K_{p l}$ are the velocity and position feedback gain matrices. They are assumed to be diagonal matrices with positive elements. This control considers only viscous friction compensation $C \dot{x}$. From Eqs. (3) and (4), we propose the following contouring control with the Coulomb friction term:

$$
\begin{aligned}
v= & M\left\{\ddot{r}-R\left(K_{v l} \dot{e}_{l}+K_{p l} e_{l}\right)-\ddot{\theta} I_{e} e_{w}+\dot{\theta}^{2} I e_{w}-\right. \\
& \left.2 \dot{\theta} I_{e} e_{w}\right\}+\eta_{1} \operatorname{sgn}(\dot{x})+\eta_{2} \dot{x} \\
\eta_{1}= & \operatorname{diag}\left\{\eta_{i 1}\right\}, \\
\eta_{2}= & \operatorname{diag}\left\{\eta_{i 2}\right\}, \quad i=1,2
\end{aligned}
$$

where $\eta_{i 1}(\geq 0)$ and $\eta_{i 2}(\geq 0)$ are the Coulomb friction force and viscous friction coefficient on the axis $i$, respectively. 


\section{Experiment}

\subsection{Circular contouring control for estimating friction coefficients}

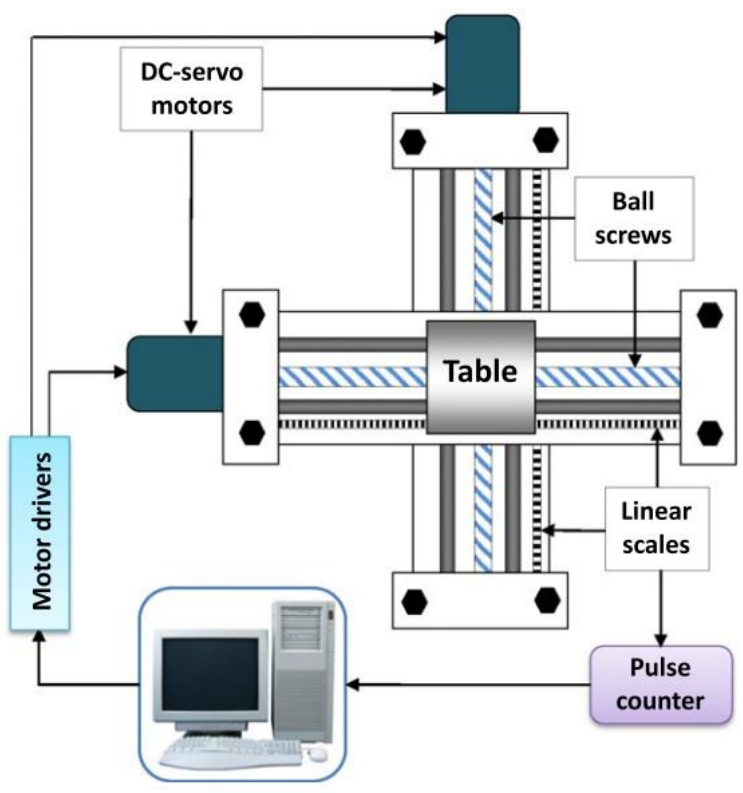

Fig. 2: Experimental setup.

The contouring controller in Eq. (4) was first applied to an $\mathrm{X} 1-\mathrm{X} 2$ table driven by $\mathrm{DC}$ servomotors and ball screw drives as shown in Fig. 2. The X1-X2 table position is measured by rotary encoders of servomotors whose resolution for position measurement is $0.025 \mu \mathrm{m}$. The velocity of each drive axis is calculated by the backward difference operation of position measurement. Sampling period is $5 \mathrm{~ms}$. We have the following parameter values of the experiment system:

$$
\begin{aligned}
& m_{1}=0.045 \mathrm{Vs}^{2} / \mathrm{mm} ; m_{2}=0.061 \mathrm{Vs}^{2} / \mathrm{mm} \\
& c_{1}=0.21 \mathrm{Vs} / \mathrm{mm} ; c_{2}=0.24 \mathrm{Vs} / \mathrm{mm}
\end{aligned}
$$

and controller gains are set as follows:

$$
\begin{aligned}
& K_{v l}=2 \omega, K_{p l}=\omega^{2}, \omega=\operatorname{diag}\left\{\omega_{l i}\right\}, \mathrm{i}=1,2, \\
& \omega_{l 1}=40 \mathrm{rad} / \mathrm{s}, \omega_{l 2}=60 \mathrm{rad} / \mathrm{s}
\end{aligned}
$$

The following reference trajectories with speed $\pi \mathrm{mm} / \mathrm{s}$ are used for the experiments:

$$
\begin{aligned}
& r_{1}=5 \cos \frac{2 \pi}{10} t \mathrm{~mm} . \\
& r_{2}=5 \sin \frac{2 \pi}{10} t \mathrm{~mm} .
\end{aligned}
$$

Equation (4) is rewritten as follows for the friction analysis:

$$
\begin{gathered}
v=\alpha+\delta \\
\alpha=\left[\alpha_{1}, \alpha_{2}\right]^{T}=M \ddot{r}+C \dot{x}, \\
\delta=\left[\alpha_{1}, \alpha_{2}\right]^{T}=M\left\{-R\left(K_{v l} \dot{e}_{l}+K_{p l} e_{l}\right)-\ddot{\theta} I_{e} e_{w}+\dot{\theta}^{2} I e_{w}\right. \\
\left.\quad-\quad 2 \dot{\theta} I_{e} e_{w}\right\}
\end{gathered}
$$

Hereafter, we call $\alpha$ and $\delta$ are a nominal dynamics term and a control term, respectively. With $\omega_{l 1}=40 \mathrm{rad} / \mathrm{s}$, $\omega_{l 2}=60 \mathrm{rad} / \mathrm{s}$. We have the following results of the experiment:

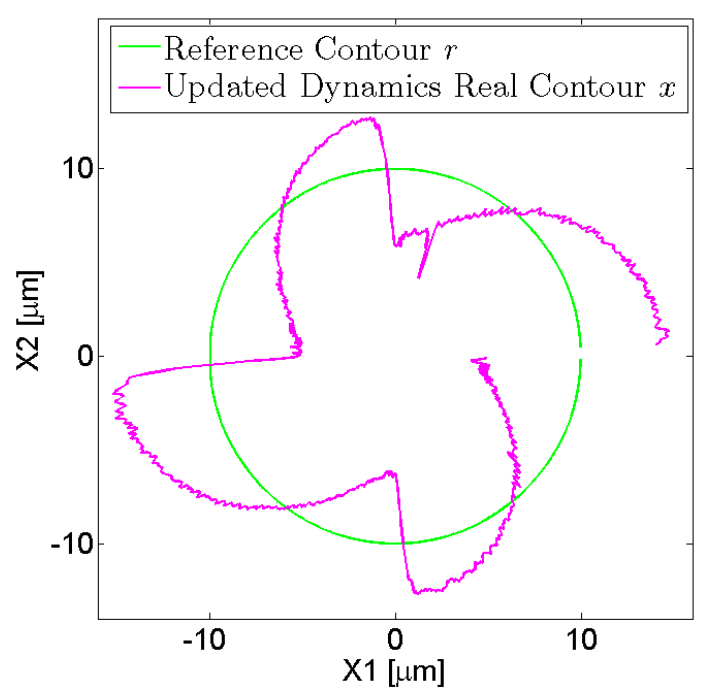

Fig. 3: Contour profile in conventional control with circular contour error enlarged by 400 times, $R=5 \mathrm{~mm}$, Speed $\pi \mathrm{mm} / \mathrm{s}$.

The profile of the contour error in the X1-X2 plane is shown in Fig. 3, in which the magnitude of the contour errors is enlarged by 400 times and the significant contour error is confirmed.

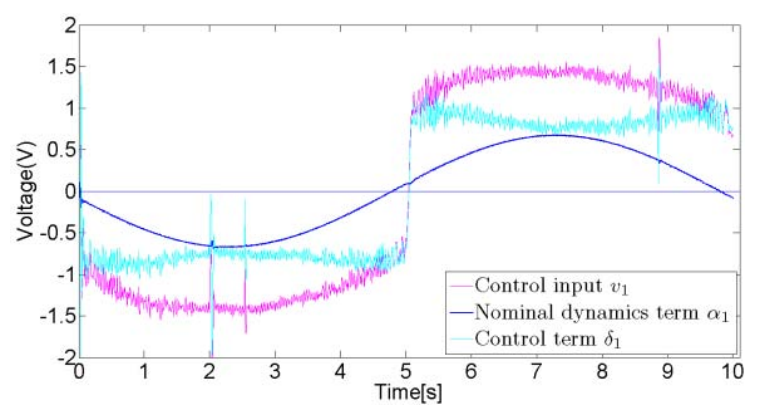

(a) X1 axis

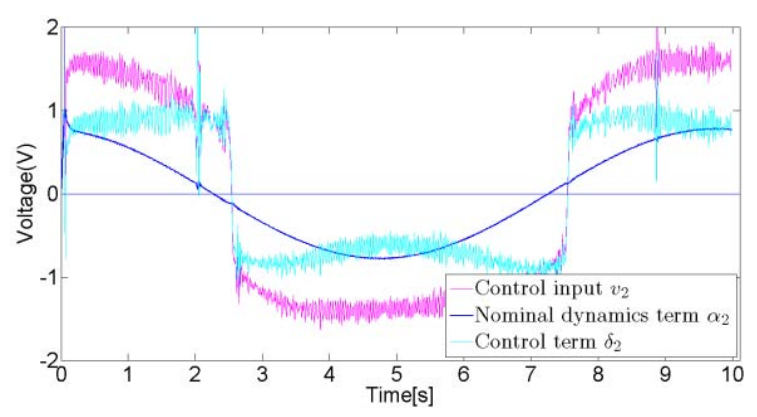

(b) X2 axis

Fig. 4: Control input profiles in conventional control.

Control input profiles in conventional control are shown in Fig. 4. Note that $\delta_{i} \approx 0$ when tracking error is close to zero in Eq. (3), and then $\alpha_{i} \approx v_{i}$ is obtained. In this case, however, $\alpha_{i}$ does not match $v_{i}$ because the nominal dynamics is very different from the actual one due to friction. 


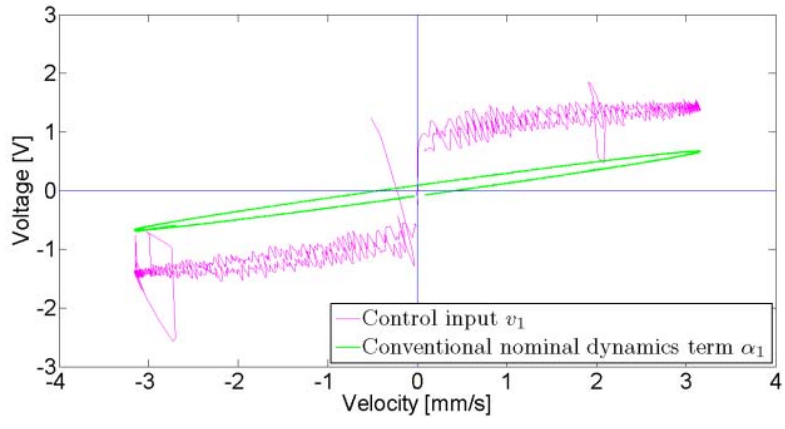

Fig. 5: Relation between control input and velocity on $\mathrm{X} 1$ axis.

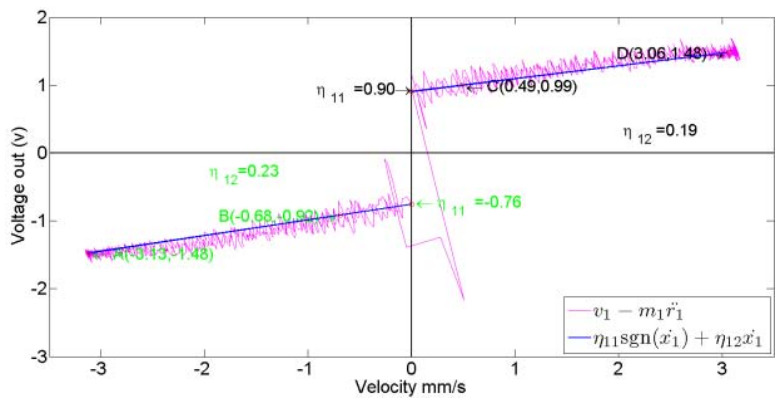

(a) Estimation of friction property

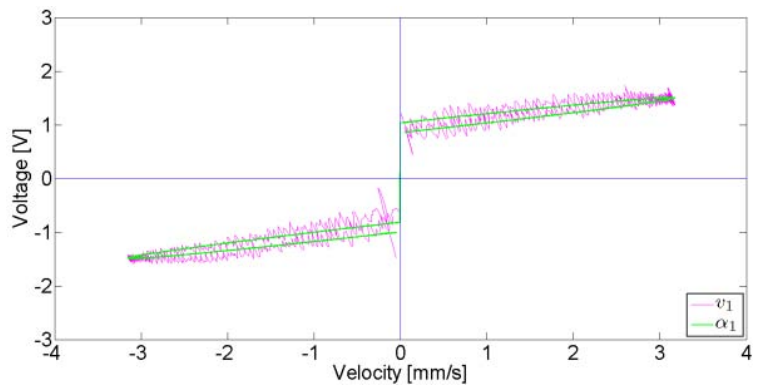

(b) Comparison between control input and nominal dynamics

Fig. 6: Friction coefficient estimation for $\mathrm{X} 1$ axis.

Next we consider friction parameter estimation. Figure 5 shows the relation between the velocity and the control input. In Fig. 6a, the term $v_{1}-m_{1} \ddot{r_{1}}$ is regarded as the disturbance compensation control. This property can be approximated by linear functions with respect to positive and negative velocities. Therefore, the friction $\eta_{11} \operatorname{sgn}(\dot{x})+\eta_{12} \dot{x}$ is obtained approximately as shown in Fig. 6a. The values of $\eta_{11}$ and $\eta_{12}$ correspond to an intersection with the vertical axis and inclination of the graph. Figure $6 \mathrm{~b}$ shows the comparison between control input $v_{1}$ and nominal dynamics term $\alpha_{1}$, in which $\alpha_{1}$ is changed as $\alpha_{1}=m_{1} r_{1} \cdot \eta_{11} \operatorname{sgn}\left(\dot{x_{1}}\right)+\eta_{12} \dot{x_{1}}$. Applying the same method to X2 axis, we have the following values for friction that depend on the sign of $\dot{x}_{1}$ and $\dot{x}_{2}$ :

$\eta_{11}=0.90 \mathrm{~V}, \eta_{12}=0.19 \mathrm{Vs} / \mathrm{mm}$, when $\dot{x}_{1} \geq 0$

$\eta_{11}=0.76 \mathrm{~V}, \eta_{12}=0.23 \mathrm{Vs} / \mathrm{mm}$, when $\dot{x}_{1}<0$

$\eta_{21}=0.67 \mathrm{~V}, \eta_{22}=0.31 \mathrm{Vs} / \mathrm{mm}$, when $\dot{x}_{1} \geq 0$

$\eta_{21}=0.83 \mathrm{~V}, \eta_{22}=0.19 \mathrm{Vs} / \mathrm{mm}$, when $\dot{x}_{1}<0$

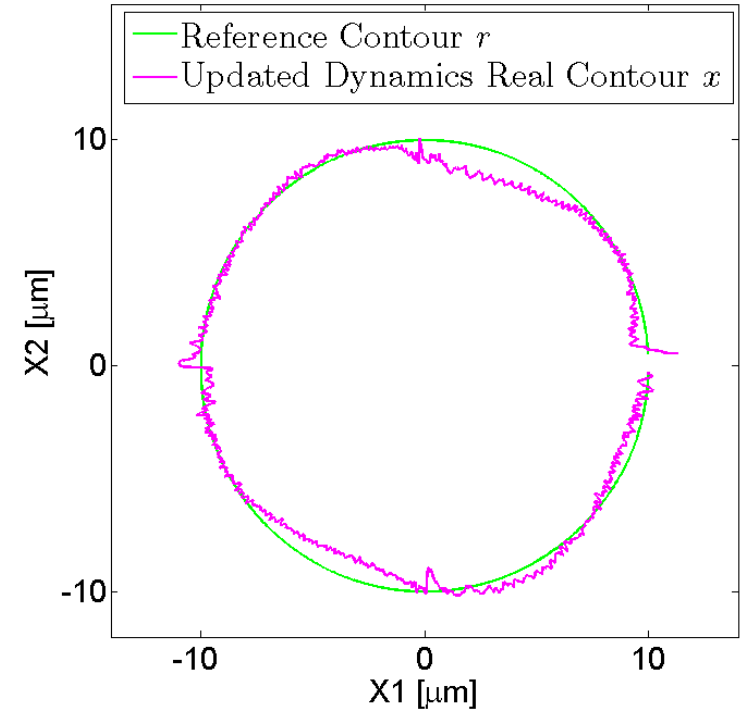

Fig. 7: Contour error profile in proposed control

$(R=5 \mathrm{~mm}$, Speed $3.14 \mathrm{~mm} / \mathrm{s})$.

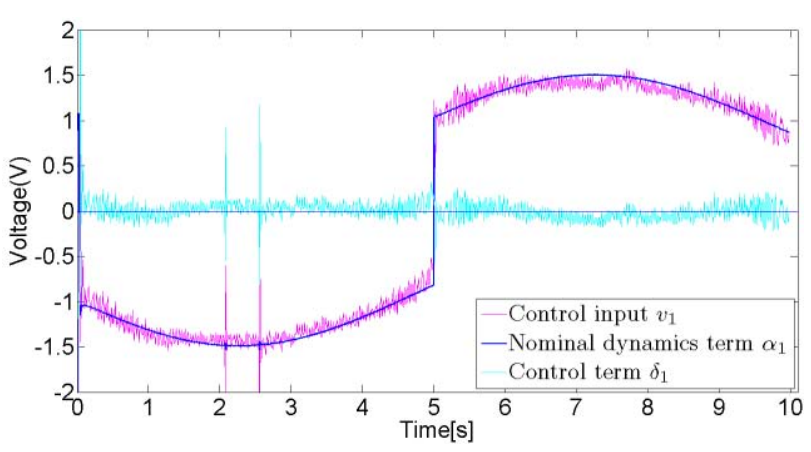

(a) $\mathrm{X} 1$ axis

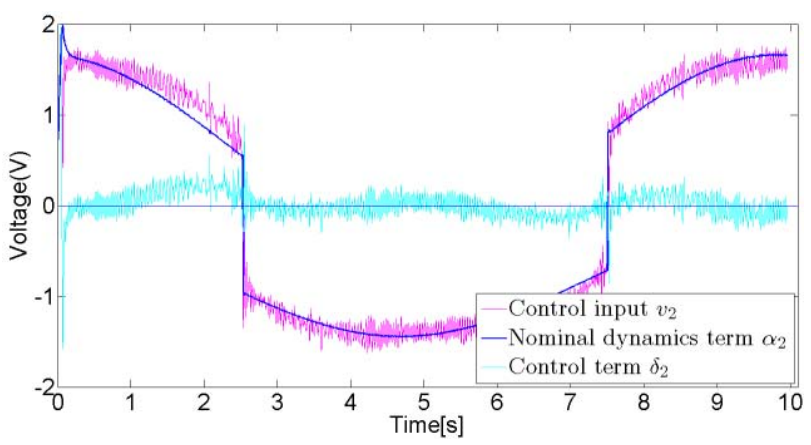

(b) X2 axis

Fig. 8: Control input profiles in proposed control.

After applying Eq. (5) with these parameters, we obtain the result in Fig. 7, in which $\alpha$ is changed as $\alpha=M \ddot{r}+\eta_{1} \operatorname{sgn}(\dot{x})+\eta_{2} \dot{x}$. The contouring error is significantly reduced. The control input profile and its $\alpha_{i}$ and $\delta_{i}$ components are shown in Fig. 8. Because the tracking error is very small, we have $\delta_{i} \approx 0$ for both drive axes. This concludes that applying simple friction compensation considering the Coulomb and viscous one achieves the ideal motion state and improves control performance significantly. 


\subsection{Experimental results for several contour curves}

We compared the proposed controller with friction compensation with conventional one by applying them to different contour curves in different speeds.

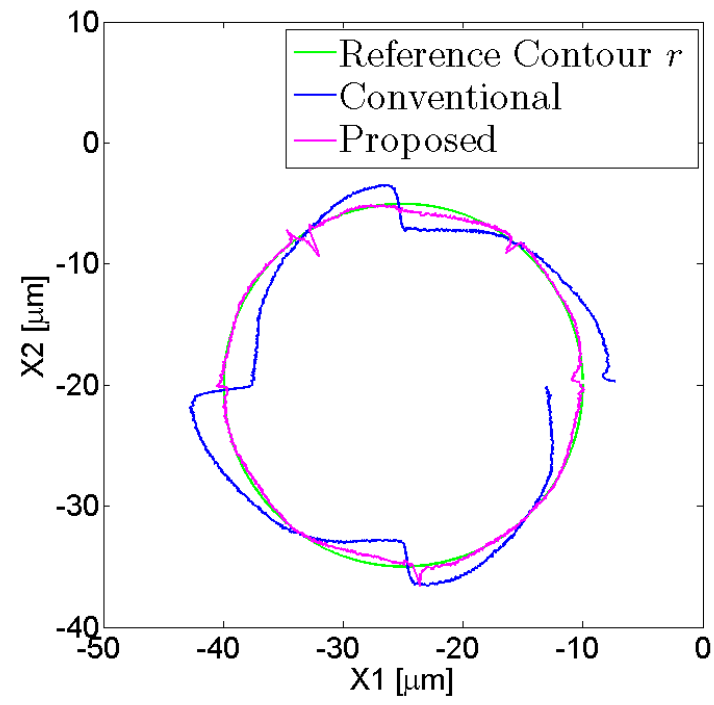

(a) Contour error profile

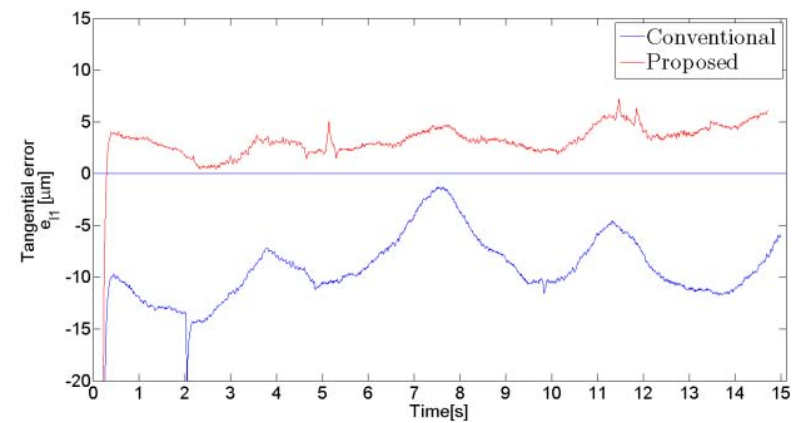

(b) Tangential error

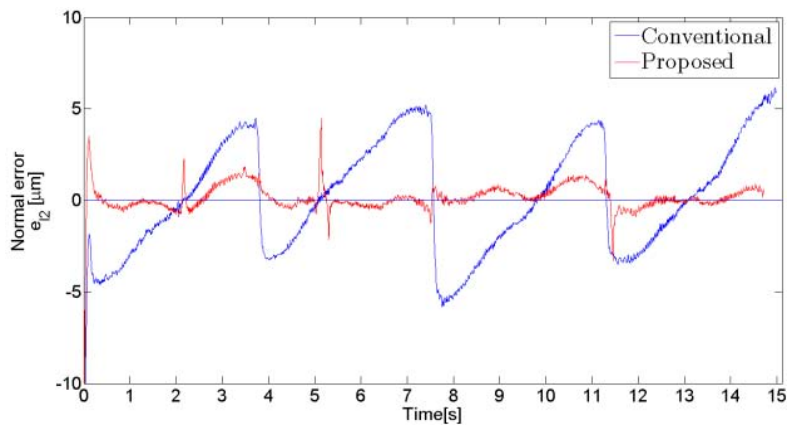

(c) Normal error

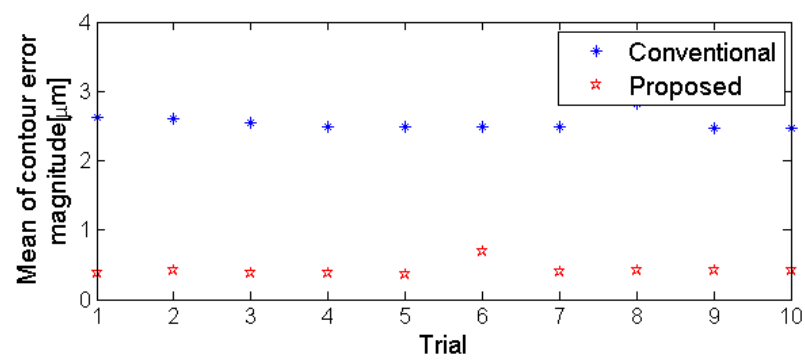

(d) 10 times trial result

Fig. 9: Circular contour $(R=15 \mathrm{~mm}$, Speed $6.28 \mathrm{~mm} / \mathrm{s})$.
Figure 9a shows the comparison results of conventional controller and proposed controller. Figure $9 \mathrm{~b}$ and Figure $9 \mathrm{c}$ show the tangential and normal errors to the contour, in which the proposed method reduced the normal error significantly. Figure 9d is the contour error magnitude for 10 times trial, and repeatability is confirmed.

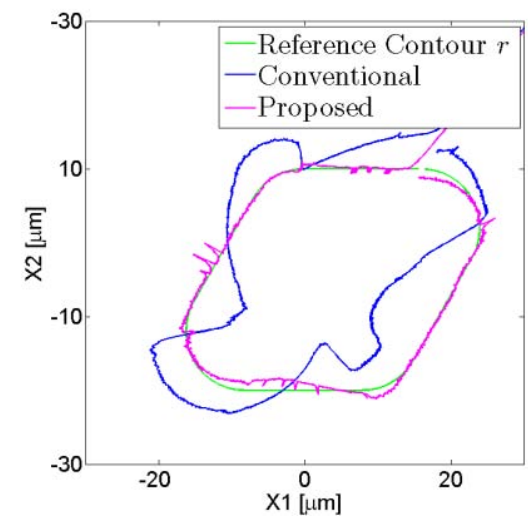

(a) Contour error profile

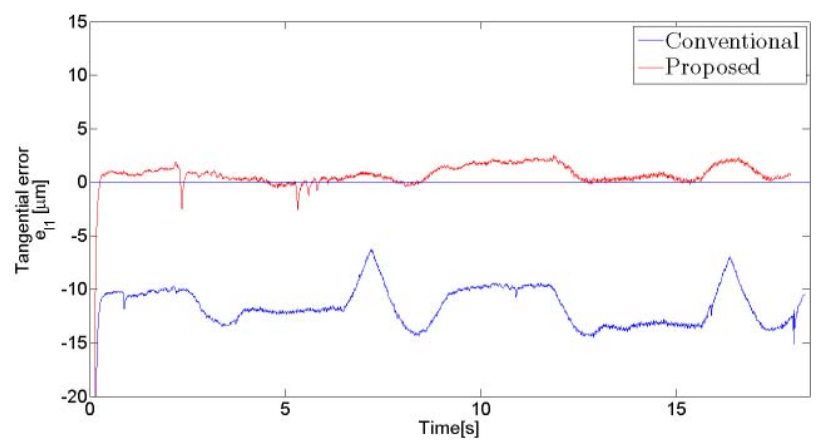

(b) Tangential error

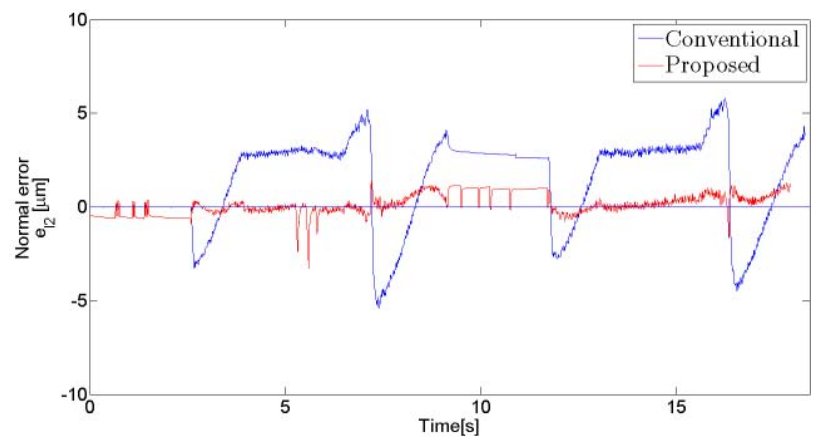

(c) Normal error

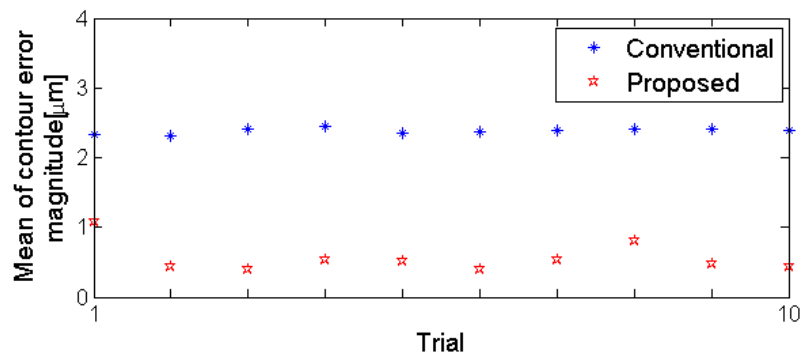

(d) 10 times trial result

Fig. 10: Parallelogram Contour (Speed 3.14mm/s). 


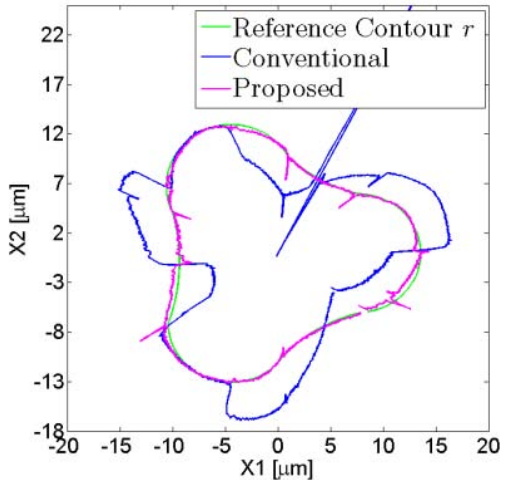

(a) Contour error profile

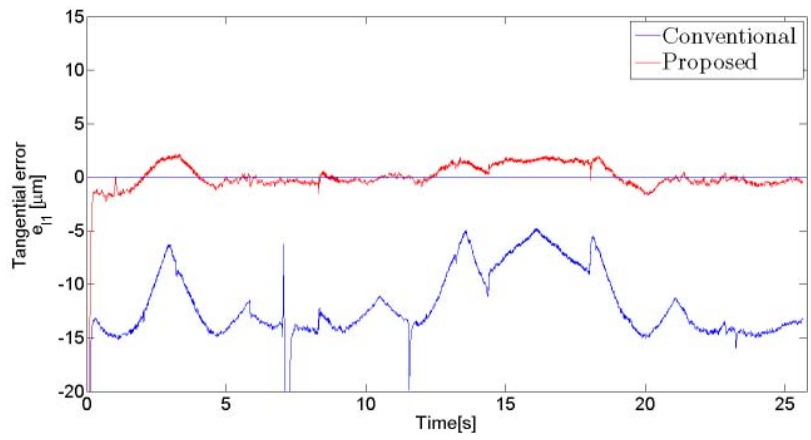

(b) Tangential error

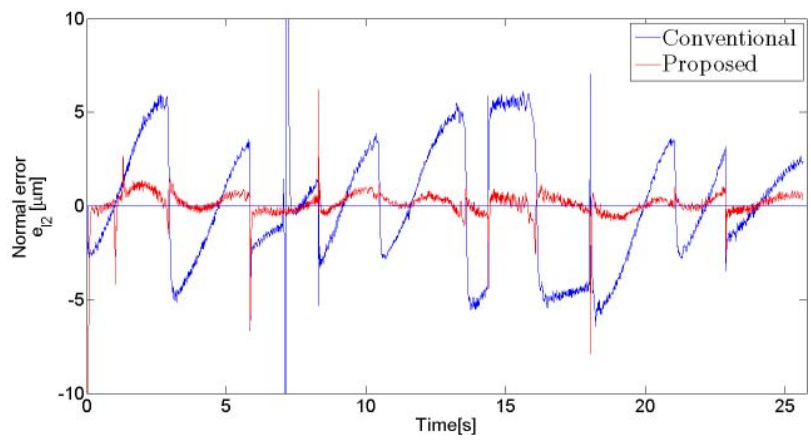

(c) Normal error

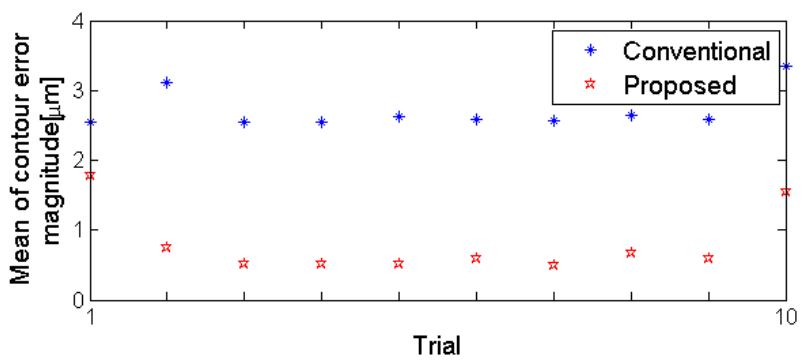

(d) 10 times trial result

Fig. 11: Triangle Contour (Speed 3.14mm/s).

Figures 10 and 11 show comparison results for noncircular contour curves by using the same control parameters with those in Fig. 9. The meaning of each figure (a)-(d) is the same as those in Fig. 9. This result confirms the effectiveness of proposed method, which gives a simple way for improving the contouring performance of feed drive systems.

\section{Conclusion}

Using the circular contouring control for friction analysis of biaxial feed drive dynamics and enhancement of contouring performance by friction compensation have been considered in this study. Experiments of applying to different curves in different speeds confirm the effectiveness of the proposed controller.

\section{Acknowledgement}

This work was supported by JSPS KAKENHI grant number 24560131.

\section{References}

[1] R. Ramesh, M. A. Mannan, and A. N. Poo, "Tracking and Contour Error Control in CNC Servo Systems", Int. Journal of Machine Tools and Manufacture, 45, pp. 301-326, 2005.

[2] Y. Koren, "Cross-Coupled Biaxial Control for Manufacturing Systems", ASME Journal of Dynamic Systems, Measurement, and Control, 102, pp. 265-272, 1980.

[3] P. K. Kulkarni and K. Srinivasan, "Optimal Contouring Control of Multi-Axis Feed Frive Servomechanism", ASME Journal of Engi- neering for Industry, 111, pp. 140-148, 1989.

[4] S.-L. Chen and K.-C. Wu, "Contouring Control of Smooth Paths for Multiaxis Motion Systems Based on Equivalent Errors", IEEE Transactions on Control System Technology, 15-6, pp. 1151-1158, 2007.

[5] C.-C. Lo and C.-Y Chung, "Tangential Contouring Controller for Biaxial Motion Control", ASME Journal of Dynamic Systems, Measurement, and Control, 121, pp. 126-129, 1999.

[6] G. T.-C. Chiu and M. Tomizuka, "Contouring Control of Machine Tool Feed Drive Systems: A Task Coordinate Frame Approach", IEEE Transactions on Control System Technology, 9-1, pp. 130- 139, 2001.

[7] X. Ye, X. Chen, X. Li, and S. Huang, "Cross-Coupled Path Precompensation Algorithm for Rapid Prototyping and Manufacturing", Int. Journal of Advanced Manufacturing Technology, 20, pp. 39-43, 2002.

[8] N. Uchiyama, T. Nakamura, and K. Yamazaki, "Reduction of Consumed Energy and Control Input Variance in Machine Tool Feed Drives by Contouring Control". International Journal of Automation Technology, Vol.3, No.4 pp. 401-407, 2009.

[9] E. Papadopoulos and G. Chasparis, "Analysis and Model-based Control of Servomechanisms with Friction". ASME J. Dynamic Systems, Measurement and Control, vol.126-4, pp.911-915, 2004.

[10] C. Canudas de Wit, H. Olsson, K.J. Astrom, and P. Lischinsky, " A New Model for Control of Systems with Friction". IEEE Transaction on Automatic Control, vol.40-3, pp.419-25, 1995. 